\section{Associação glicemia de jejum e fatores de risco como teste para rastreamento do diabete gestacional}

\section{Fasting glycemia associated with risk factors as a screening test for gestational diabetes mellitus}

Wilson Ayach 1

Iracema de Mattos Paranhos Calderon 2

Marilza Vieira Cunha Rudge 3

Roberto Antonio Araújo Costa 3

1 Departamento de Gineco-Obstetrícia. Universidade Federal de Mato Grosso do Sul. Rua Filinto Muller, s. n. Cidade Universitária. Campo Grande, MS, Brasil. CEP: 79.080-190 E-mail: wilsonayach@uol.com.br

2-3 Departamento de Ginecologia e Obstetrícia. Faculdade de Medicina de Botucatu. Universidade Estadual Paulista. Botucatu, SP, Brasil

\section{Resumo}

Objetivos: comparar dois testes de rastreamento do diabete gestacional.

Métodos: estudo prospectivo no qual foram avaliadas 356 gestantes, sem diagnóstico prévio do diabete melito, submetidas, de modo independente, a dois testes de rastreamento: associação glicemia de jejum e fator de risco $(G J+F R)$ e teste oral simplificado de tolerância à glicose (TTG50g). A comparação entre os métodos foi realizada pelos índices de sensibilidade $(S)$, especificidade (E) e valores preditivos positivo (VPP) e negativo $(V P N)$, resultados falsos, positivos (FP) e negativos (FN) e pela diferença dos resultados observados e esperados, avaliada pelo teste do Qui-quadrado $(p<0,05)$.

Resultados: a associação $G J+F R$ determinou a confirmação diagnóstica em maior número de gestantes (187; 52,5\%) que o TTG50g (49; 13,8\%). Esta diferença foi significativa $(p<0,05)$. A associação $G J+F R$ apresentou sensibilidade de $83,7 \%$ e valor preditivo negativo (VPN) de 95,3\% em relação ao TTG50g.

Conclusões: os índices elevados de sensibilidade e $V P N$ da associação GJ+FR em relação ao TTG50g, sua simplicidade, praticidade, baixo custo e fácil replicação permitem sua indicação no rastreamento do diabete gestacional.

Palavras-chave Diabete gestacional, Glicemia, Fatores de risco, Teste de tolerância à glicose

Key words Diabetes, gestacional, Blood glucose, Risk factors, Glucose tolerance test 


\section{Introdução}

A gravidez caracteriza-se pelo aumento progressivo das necessidades e da resistência à insulina. $\mathrm{O}$ estresse hormonal da gestação, observado em mulheres e comprovado em animais, ${ }^{1}$ testa a função das ilhotas de Langerhans. Nas mulheres com deficiência parcial ou completa na função das ilhotas de Langerhans, ocorre a intolerância à glicose, franca ou limítrofe. Essa intolerância aos carboidratos, de qualquer gravidade, quando diagnosticada durante a gravidez é denominada diabete gestacional. ${ }^{2}$

O diagnóstico dessa alteração metabólica previne riscos maternos e fetais. A mãe é alertada sobre uma condição que pode afetar sua saúde no futuro e os riscos fetais, associados à tolerância diminuída à glicose, podem ser prevenidos ou minimizados com o tratamento adequado. 3,4

Não existe, contudo, consenso a respeito dos métodos de rastreamento e diagnóstico do diabete gestacional. Isso porque essa afecção específica aglutina uma série de distúrbios relacionados à ação e à secreção de insulina e seu simples reconhecimento, pela primeira vez na gravidez, não descarta a possibilidade de sua pré-existência. ${ }^{1} \mathrm{O}$ teste de rastreamento, que seleciona as gestantes para o teste de confirmação diagnóstica, deve ser preciso, de fácil execução, de baixo custo e passível de reprodução. 5

O teste oral de tolerância à glicose simplificado com sobrecarga de 50g (TTG50g), independente do estado alimentar da gestante, consiste na dosagem da glicemia plasmática uma hora após sobrecarga oral de $50 \mathrm{~g}$ de glicose. É o teste de rastreamento mais utilizado no mundo, provavelmente pela alta correlação com o teste de tolerância à glicose com sobrecarga de 100g (TTG100g), ${ }^{6}$ pela capacidade de predizer o desenvolvimento do diabete melito futuro $^{7}$ e a recorrência de diabete gestacional. ${ }^{8}$ O'Sullivan et al.,6 recomendam sua realização em todas as gestantes, entre a $24^{\mathrm{a}}$ e a $28^{\mathrm{a}}$ semanas e, consideram $140 \mathrm{mg} / \mathrm{dL}$ como ponto de corte. Considerando esse limite, os autores encontraram sensibilidade de $78 \%$ e especificidade de $87 \%$ em relação ao TTG100g. Diminuindo o limite para $130 \mathrm{mg} / \mathrm{dl}$, a sensibilidade elevou-se para $100 \%$, sem alteração significativa da especificidade. 5 O TTG50g é, entretanto, um teste que exige disponibilidade de tempo e material para sua execução, além de causar certo desconforto para a gestante.

A Organização Mundial da Saúde (OMS) não diferencia o rastreamento e diagnóstico do diabete melito, na gestação ou fora dela. Preconiza o teste de tolerância à glicose com sobrecarga de $75 \mathrm{~g}$
(TTG75g) em todas as gestantes, como único teste, tanto para rastreamento como para diagnóstico do diabete na gestação. Consiste na dosagem das glicemias plasmáticas no jejum e duas horas após a sobrecarga oral de glicose. Além de apresentar todas as restrições referidas em relação ao TTG50g, esse protocolo exige maior tempo, o que poderia dificultar sua realização universal para o rastreamento do diabete gestacional.

A glicemia materna, no jejum ou casual, pode ser empregada no rastreamento do diabetes gestacional. A glicemia casual, independente do tempo decorrido desde a última refeição, não apresenta boa correlação com os testes de tolerância à glicose e, por isso, não é muito utilizada. ${ }^{9}$

A glicemia de jejum, muito utilizada fora da gestação, pela facilidade de execução, baixo custo e conforto para o paciente, 10 tem sido, inexplicavelmente, pouco utilizada no rastreamento de gestantes. Sua acurácia em relação ao TTG $100 \mathrm{~g}$, fora da gravidez, varia com o valor de corte empregado. Para um limite de $100 \mathrm{mg} / \mathrm{dl}$, a sensibilidade é de $90,7 \%$, a especificidade de $83,5 \%$, o valor preditivo positivo de $39,7 \%$ e o valor preditivo negativo de $98,7 \% .11$

A acurácia da glicemia de jejum no rastreamento do diabete gestacional, a exemplo do TTG50g, varia com o valor de corte empregado e com a população estudada. Para um limite de $119 \mathrm{mg} / \mathrm{dl}$, West et al. 12 encontraram sensibilidade de $23,0 \%$ na Venezuela, $63,6 \%$ no Uruguai e $100 \%$ na Malásia. Sacks et al. 13 encontraram sensibilidade de $80,0 \%$ e especificidade de $40,0 \%$ para um limite de $88 \mathrm{mg} / \mathrm{dl}$, o que já referenda sua utilização no rastreamento do diabetes gestacional. Além disso, apresenta boa correlação com o prognóstico materno e fetal, visto que gestantes com glicemia no jejum maior que $105 \mathrm{mg} / \mathrm{dl}$ têm risco de macrossomia treze vezes maior, quando comparadas a gestantes com glicemia de jejum menor que $85 \mathrm{mg} / \mathrm{dl} .14$

A prevalência do diabete gestacional está diretamente relacionada ao valor da glicemia de jejum, sendo de $12,0 \%$ nas pacientes com glicemia de jejum $90 \mathrm{mg} / \mathrm{dl}$ e de $0,8 \%$ naquelas com jejum inferior a $90 \mathrm{mg} / \mathrm{dl} .15$ A prevalência de diabete melito em gestação futura, 16 bem como do diabetes melito tipo 2 no futuro da vida da mulher foram mais freqüentes nas gestantes com glicemia de jejum alterada. 8,16 Entretanto, a correlação da glicemia de jejum e recorrência do diabete gestacional não foi confirmada por Moses. 17

Durante a gestação, a glicemia de jejum permanece relativamente estável, porém é mais baixa no primeiro trimestre. Assim, valores suposta- 
mente normais ou discretamente elevados (90mg/dl) já representariam fator de risco para o diabete gestacional.

Guttorm 15 recomenda a dosagem da glicemia de jejum na primeira metade da gravidez como teste de rastreamento, utilizando $90 \mathrm{mg} / \mathrm{dl}$ como valor de corte, pois não encontrou nenhum caso de diabete gestacional onde a glicemia de jejum da primeira metade da gravidez fosse inferior a $90 \mathrm{mg} / \mathrm{dl}$ ou não apresentasse fator de risco. 15 Outros autores reforçam esse ponto de corte evidenciando que a glicemia de jejum é maior que $90 \mathrm{mg} / \mathrm{dl}$ em apenas $2 \%$ das gestantes não diabéticas. $5,6,18$

No Brasil, um estudo multicêntrico, utilizando os critérios da OMS como padrão diagnóstico, demonstrou que a sensibilidade e a especificidade da glicemia de jejum variaram com o valor de corte empregado. O melhor resultado do teste foi observado no limite de $85 \mathrm{mg} / \mathrm{dl}$, com sensibilidade de $94,0 \%$ e especificidade de $66,0 \% .19$

Não obstante todas essas vantagens, a glicemia de jejum é pouco utilizada como teste de rastreamento do diabete gestacional. Bertini-Oliveira et al.20 argumentam que, apesar da hipoglicemia materna do primeiro trimestre da gestação, a média da glicemia de jejum $(96 \mathrm{mg} / \mathrm{dl})$ encontrada nas gestantes diabéticas está dentro dos limites de normalidade. Assim, a glicemia de jejum como teste de rastreamento do diabete gestacional resultaria em perda de 39,0\% dos casos. 21 Além disso, outrora, o diagnóstico do diabete gestacional era baseado na normalidade da glicemia de jejum e na hiperglicemia no período pós-prandial, o que desestimulou seu emprego no rastreamento do diabete gestacional. A glicemia de jejum apresenta, ainda, outros pontos desfavoráveis ao seu emprego, como o fato de nem sempre as pacientes apresentarem-se em jejum, de oito a doze horas, para o exame e a dificuldade de se encontrar um valor de corte confiável pela curva ROC.

A dificuldade em encontrar o teste de rastreamento ideal acaba valorizando o mais simples e antigo - a história clínica - que identifica os fatores de risco para o diabete gestacional. A presença de um ou mais dos fatores de risco, entre eles, antecedente familiar de diabete melito, idade igual ou superior a 30 anos, 22 obesidade, antecedente de diabete gestacional, glicosúria, óbito fetal sem causa aparente, malformação e macrossomia fetal, identifica, de forma incontestável, um grupo de alto risco para o desenvolvimento do diabete gestacional. 23

Os fatores de risco estão presentes em grande proporção de indivíduos de uma população, 6,24 por isso, devem evidenciar a maioria absoluta dos doentes; entretanto, podem estar presentes, também, em indivíduos completamente normais. ${ }^{25}$ Sacks et al.,24 encontraram fatores de risco em $77,0 \%$ das gestantes, quando se considerou idade materna superior a 25 anos e peso materno igual ou superior a $68 \mathrm{~kg}$. Ao excluir esses fatores, encontraram fator de risco em apenas $55,0 \%$ dessas gestantes. A incidência de TTG50g alterado e de diabete gestacional também é significativamente maior no grupo de mulheres que apresentam fatores de risco.23,26 Além disso, as diabéticas gestacionais têm maior prevalência de fatores de risco. 26,27

Weeks et al. ${ }^{28}$ demonstraram que a presença de fatores de risco não influenciou no prognóstico fetal, pois não encontraram diferença significativa na incidência de cesárea, macrossomia, distocia de bisacromial e necessidade de insulinoterapia entre gestantes diabéticas, independente de terem, ou não, fatores de risco. Por outro lado, o diabete gestacional não é doença exclusiva de mulheres com fatores de risco e pode ser encontrado em gestantes sem qualquer estigma da doença. Das pacientes com diagnóstico de diabete gestacional, 39,0\% não apresentavam qualquer fator de risco. 3,25

A associação glicemia de jejum e fator de risco (GJ+FR) tem sido utilizada para o rastreamento do diabete gestacional.29 $\mathrm{O}$ rastreamento é considerado positivo quando a glicemia de jejum for igual ou superior a $90 \mathrm{mg} / \mathrm{dl}$, isolada ou associada à presença de antecedentes familiares, pessoais ou obstétricos relacionados ao diabete melito e gestacional. No Brasil, Rudge et al. 30 submeteram 782 gestantes com esse tipo de rastreamento (GJ+FR) positivo, ao TTG50g e verificaram concordância dos resultados. A glicemia de jejum isolada, igual ou superior a $90 \mathrm{mg} / \mathrm{dl}$ foi encontrada em $22,0 \%$ dos casos e, o TTG50g igual ou superior a $140 \mathrm{mg} / \mathrm{dl}$, em $17,5 \%$ das gestantes. Se, além do TTG50g, o fator de risco, já corrigido, fosse incluído no critério proposto por O'Sullivan et al.,6 os métodos de rastreamento seriam equivalentes, indicando a mesma proporção de gestantes para a confirmação diagnóstica. Entretanto, não se sabe se o TTG50g e a glicemia de jejum estavam alterados nas mesmas gestantes e, também, se os resultados seriam semelhantes mesmo nas gestantes sem fator de risco e com glicemia de jejum menor que $90 \mathrm{mg} / \mathrm{dl}$. Dessa forma, para sua validação, é necessária a comparação com os testes de rastreamento (TTG50g) e diagnóstico (TTG100g) mais utilizados. O presente estudo refere-se, portanto à apresentação de resultados parciais da comparação entre esses testes.

Diante de tais aspectos, é necessário validar associação glicemia de jejum e fator de risco como 
teste de rastreamento do diabete gestacional. O objetivo geral do trabalho foi comparar dois testes de rastreamento - associação GJ+FR e TTG50g e especificamente calcular os valores de sensibilidade, especificidade, valores preditivos positivo e negativo e resultados falsos positivo e negativo da associação GJ+FR em relação ao TTG50g.

\section{Métodos}

A pesquisa foi conduzida na Maternidade do Núcleo do Hospital Universitário da Universidade Federal de Mato Grosso do Sul, (NHU/UFMS), Brasil, no período de outubro de 1997 a fevereiro de 1999 . Trata-se de estudo de validação de testes disgnósticos, para comparação de dois testes de rastreamento do diabete gestacional: - a associação glicemia de jejum e fator de risco (GJ+FR) e o teste oral simplificado de tolerância à glicose (TTG50g), aplicados de forma independente na mesma população de gestantes. Para o cálculo do tamanho amostral mínimo, considerou-se a população finita, 12000 partos na cidade de Campo Grande, Mato Grosso do Sul, a incidência prévia do diabete gestacional como variável nominal $(5,0 \%)$ e o erro amostral de $2,5 \%$. O valor calculado foi 347 e foram analisadas 356 gestantes. 30

Foram consideradas elegíveis para participar do estudo todas as gestantes, sem diagnóstico prévio de diabete melito, que procuraram o ambulatório de pré-natal do NHU/UFMS, antes da $20^{\mathrm{a}}$ semana de gravidez. Foram estipulados como critérios de descontinuidade a desistência voluntária do estudo, a não-realização de qualquer um dos testes de rastreamento e a interrupção da gestação antes de se completar o protocolo de pesquisa.

Todas as gestantes que preencheram os critérios de inclusão, após esclarecimento do projeto e aceite em participar, assinaram o termo de consentimento livre e esclarecido. Em seguida, foram introduzidas no seguinte protocolo - anamnese, determinação da idade gestacional, dosagem plasmática de glicose de jejum e teste oral simplificado de tolerância à glicose (TTG50g).

A anamnese foi realizada na primeira consulta da assistência pré-natal, visando identificar os fatores de risco, preconizados por Rudge e De Luca 29 e pelo Colégio Americano de Ginecologia e Obstetrícia (ACOG). Foram eles: idade igual ou superior a 30 anos, índice de massa corporal (IMC) $\geq 27 \mathrm{~kg} / \mathrm{m} 2$, antecedente pessoal de diabete gestacional, antecedente familiar de diabete melito, macrossomia, óbito fetal sem causa aparente, aborta- mento habitual e malformação fetal em gestação anterior. A idade gestacional foi determinada pela regra de Näegele e confirmada por ultra-sonografia na primeira metade da gestação.

A análise dos níveis de glicose plasmática de jejum foi realizada antes da $20^{\mathrm{a}}$ semana de gestação, às sete horas da manhã, após jejum de 8 a 12 horas, conforme padronização do Ministério da Saúde. Para o TTG50g seguiu-se a última recomendação da American Diabetes Association (ADA). ${ }^{2} \mathrm{O}$ teste foi aplicado entre a $24^{\mathrm{a}}$ e a $28^{\mathrm{a}}$ semanas e constou da ingestão de $50 \mathrm{~g}$ de glicose (55g de dextrosol) diluídas em $200 \mathrm{ml}$ de água, com a paciente livre de jejum e orientada para repouso e abstinência de líquidos e sólidos durante o exame. As glicemias, de jejum e de uma hora após a sobrecarga de $50 \mathrm{~g}$ de glicose, foram determinadas pelo método enzimático glicose-oxidase. 2

O rastreamento pela associação $\mathrm{GJ}+\mathrm{FR}$ foi considerado positivo nas gestantes com nível de glicose plasmática de jejum igual ou superior a $90 \mathrm{mg} / \mathrm{dl}$ e/ou na presença de qualquer fator de risco para o diabete gestacional. Para o TTG50g, considerou-se rastreamento positivo quando o valor da glicemia de uma hora foi igual ou superior a $140 \mathrm{mg} / \mathrm{dl}$.

Para a comparação das frequiências observadas e esperadas, entre os dois testes de rastreamento, utilizou-se o teste do Qui-quadrado. A acurácia da associação GJ+FR em relação ao TTG50g foi determinada pelos índices de sensibilidade, especificidade e dos valores preditivos positivo (VPP) e negativo (VPN) e taxas de resultados falsos, positivos (FP) e negativos (FN). 31

O Projeto foi aprovado pela Comissão de Pesquisa da Universidade Federal do Mato Grosso do Sul, em setembro de 1997, e referendado pelo Comitê de Ética em Pesquisa da Faculdade de Medicina de Botucatu da Universidade Estadual Paulista.

\section{Resultados}

A população foi caracterizada por $84,8 \%$ de gestantes com menos de 30 anos e por $87,6 \%$ apresentando IMC menor que $27 \mathrm{~kg} / \mathrm{m} 2$. A maioria era branca $(60,4 \%)$ e tinha mais de um filho $(54,5 \%)$ (Tabela1). A glicemia de jejum igual ou superior a $90 \mathrm{mg} / \mathrm{dl}$ foi encontrada em 48 gestantes $(13,4 \%)$, os fatores de risco estavam presentes em $168(47,2 \%)$ e o rastreamento positivo pela associação GJ+FR confirmou-se em 187 (52,5\%) do total de gestantes avaliadas. Em relação ao TTG50g, a maioria 
$(86,2 \%)$ das gestantes apresentou glicemia de uma hora pós-sobrecarga menor que $140 \mathrm{mg} / \mathrm{dl} \mathrm{e}$, em apenas $49(13,8 \%)$ confirmou-se rastreamento positivo pelo TTG50g (Tabela 2).

A aplicação dos dois métodos de rastreamento resultou em quatro tipos de respostas: - 161 gestantes $(45,2 \%)$ com os dois testes negativos, 41 $(11,5 \%)$ com os dois testes positivos, $146(41,0 \%)$ com TTG50g normal e rastreamento positivo pela associação GJ+FR e 8 (2,3\%) com TTG50g alterado e rastreamento negativo pela associação GJ+FR (Tabela 3).

O teste do Qui-quadrado evidenciou dife-rença significativa $(p<0,05)$ entre os dois métodos de rastreamento, demonstrada pelas freqüências esperadas e observadas: - a associação GJ+FR, quando positiva, encontrou maior número de TTG50g alterado e, quando negativa, maior número de TTG50g normal, em relação aos números espe-rados (Tabela 3). A comparação entre os dois testes revelou sensibilidade de $83,7 \%$, valor preditivo negativo (VPN) de 95,3\% resultado falsos-negativo de 2,3\% para a associação GJ+FR em relação ao TTG50g (Tabela 3).

Tabela 1

Características biológicas das gestantes.

\begin{tabular}{lrc}
\hline Característica & N & $\%$ \\
\hline Idade $\geq 30$ anos & 54 & 15,2 \\
IMC $\geq 27 \mathrm{~kg} / \mathrm{m} 2$ & 44 & 12,4 \\
Raça branca & 215 & 60,4 \\
Primigestas & 162 & 45,5 \\
\hline
\end{tabular}

IMC = índice de massa corporal

\section{Discussão}

A aplicação dos dois testes de rastreamento de modo independente permitiu a comparação entre a associação GJ+FR e o TTG50g. A eficácia da associação GJ+FR foi determinada em relação ao TTG50g, pois este é considerado o teste padrão para o rastreamento do diabete gestacional, o que o referenda como parâmetro de referência para validação de outro teste de rastreamento. O ideal para validação de um teste novo seria a realização do teste diagnóstico em todas as gestantes, o que nem sempre é possível. O método
Tabela 2

Distribuição de freqüência das gestantes de acordo com o método de rastreamento.

\begin{tabular}{lrr}
\hline Métodos de rastreamento & N & $\%$ \\
\hline $\begin{array}{l}\text { Glicemia } \\
\quad \text { Jejum < 90mg/dl }\end{array}$ & 308 & 86,5 \\
$\quad \geq 90 \mathrm{mg} / \mathrm{dl}$ & 48 & 13,5 \\
FR & & \\
$\quad$ Ausente & 188 & 52,8 \\
$\quad$ Presente & 168 & 47,2 \\
GJ + FR & & \\
$\quad$ Negativo & 169 & 47,5 \\
$\quad$ Positivo & 187 & 52,5 \\
TTG50g & & \\
$\quad<140 \mathrm{mg} / \mathrm{dl}$ & 307 & 86,2 \\
$\quad \geq 140 \mathrm{mg} / \mathrm{dl}$ & 49 & 13,8 \\
Total & 356 & 100,0 \\
\hline
\end{tabular}

GJ = glicemia de jejum; FR = fator de risco;

TTG50g = teste oral de tolerância à glicose simplificado.

Tabela 3

Distribuição dos resultados conforme a concordância nos dois testes de rastreamento - associação glicemia de jejum e fator de risco (GJ + FR) e teste oral de tolerância à glicose simplificado (TTG50g).

\begin{tabular}{lrrrrc}
\hline \multicolumn{5}{c}{ TTG50 $\mathbf{1 4 0} \mathbf{m g} / \mathbf{d l}$} \\
\hline Rudge e De Luca29 & Positivo Negativo & Total & $\chi^{2}$ & $\rho$ \\
\hline Positivo & 41 & 146 & 187 & 22,2 & $<0,05$ \\
Negativo & 8 & 161 & 169 & & \\
Total & 49 & 307 & 356 & & \\
\hline
\end{tabular}

Sensibilidade $=83,7 \%$; especificidade $=52,4 \%$; valor preditivo positivo $=$ $21,9 \%$; valor preditivo negativo $=95,3 \%$; falso positivos $=41,0 \%$; falso negativos $=2,3 \%$.

empregado neste trabalho não permite inferir sobre o real estado das pacientes estudadas, doentes ou não doentes, mas permite avaliar a concordância entre os testes avaliados e determinar exatamente quais são suas diferenças.

Nesse estudo, a glicemia de jejum igual ou superior a $90 \mathrm{mg} / \mathrm{dL}$ e o TTG50g alterado foram encontrados na mesma proporção de gestantes. A associação GJ+FR seleciona maior número de gestantes para a confirmação diagnóstica que o TTG50g. Isso 
se deve ao fato do rastreamento pela associação GJ+FR contemplar os fatores de risco, que, apesar de selecionarem a maioria das gestantes com TTG50g alterado, também estão presentes em muitas gestantes com TTG50g normal.

A comparação entre os dois testes avaliados foi realizada pela proporção de resultados concordantes, ambos positivos ou negativos e pela proporção de resultados discordantes. Os resultados foram concordantes em 56,7\% dos casos e a freqüência dos resultados observados e esperados confirmou diferença significativa entre os dois testes de rastreamento. 21 A associação GJ+FR, quando positiva, identificou maior número de gestantes com TTG50g alterado e, quando negativa, maior número de gestantes com TTG50g normal. Selecionou, portanto, os verdadeiros positivos e negativos. A sensibilidade da associação GJ+FR em relação ao TTG50g foi de $83,7 \%$ e o valor preditivo negativo (VPN) de 95,3\%. Esses índices indicaram baixa possibilidade do TTG50g estar alterado quando a glicemia de jejum for menor que $90 \mathrm{mg} / \mathrm{dl}$ e a gestante não apresentar fator de risco para o diabete gestacional, como demonstrado pelo baixo índice de resultados falsosnegativos.

A despeito da alta proporção de falsos-positivos evidenciada pela associação GJ+FR, a baixa incidência de falsos-negativos reforça sua condição de bom teste de rastreamento para o diabete gestacional. 5 Merece destaque a sensibilidade da GJ+FR em relação ao TTG50g. Frente às deficiências próprias do TTG50g - sensibilidade de $78 \%$ em relação ao teste mais utilizado para diagnóstico do diabete gestacional (TTG100g), 2 o índice de $83,7 \%$ seria bastante satisfatório.

Tais resultados permitem determinar qual seria o melhor teste de rastreamento do diabete gestacional? Para responder a essa pergunta alguns fatores precisam ser considerados.

O teste de rastreamento é realizado para selecionar população de risco para determinada condição, ou seja, identifica as pessoas que, apesar de não apresentarem a doença, estão sob risco de desenvolvê-la. 5 São características desejáveis aos métodos de rastreamento a elevada sensibilidade e valor preditivo negativo e os baixos índices de resultados falsos-negativos, a facilidade na execução e na reprodutibilidade, a praticidade e o conforto, além do baixo custo. 31
O método de rastreamento pela associação GJ+FR satisfaz tais condições. A anamnese, que identifica os fatores de risco, é inerente à prática médica, resgata a história clínica e aprimora a relação médico-paciente. A glicemia de jejum é exame de rotina na assistência pré-natal, de fácil reprodução e baixo custo, permitindo sua replicação sistemática em qualquer unidade de saúde do país. Desse modo, a aplicação da associação GJ+FR para o rastreamento do diabete gestacional não implicaria em custo adicional à rotina de assistência pré-natal e poderia representar até economia por dispensar o TTG50g. Por outro lado, o TTG50g representa um teste a mais na rotina da assistência pré-natal, além do desconforto na ingestão de glicose que, frente ao rastreamento positivo, deverá ser repetida durante a gestação. Implica, portanto, na adição de outro teste de sobrecarga, agora com mais três dosagens sangüíneas. ${ }^{2}$

De modo prático, a simplicidade e a praticidade da associação GJ+FR, aliadas à sua acurácia em relação ao TTG50g devem ser valorizados na ponderação do elevado número de falsos-positivos. Soma-se, ainda, a possibilidade de reprodução deste tipo de rastreamento em qualquer unidade básica de atendimento pré-natal. Considerados no total, esses argumentos reforçam sua recomendação como rastreador do diabete gestacional.

Entretanto, a exemplo do TTG50g, o rastreamento pela associação GJ+FR deverá ser comparado ao teste diagnóstico universal (TTG100g) e ao perfil glicêmico, para avaliar sua relação com o risco de diabete melito futuro e com o prognóstico da gestação atual.

\section{Conclusões}

A aplicação dos rastreamentos de Rudge e De Luca 29 e TTG50g21 na mesma população de gestantes permitiu avaliar a acurácia da glicemia de jejum associada aos fatores de risco. A sensibilidade $(83,7 \%)$ do rastreamento de Rudge e De Luca29 em relação ao TTG50g, ${ }^{21}$ associada a sua simplicidade, praticidade, baixo custo e fácil replicação em qualquer serviço de assistência pré-natal do país, permitem sua indicação no rastreamento do diabete gestacional. 


\section{Referências}

1. Calderon IMP. Influência do binômio diabete e gravidez na atividade endócrina do pâncreas materno e fetal: estudo experimental em ratas [tese doutorado]. Botucatu: Faculdade de Medicina de Botucatu da Universidade Estadual Paulista; 1994

2. ADA(American Diabetes Association). Clinical practice recommendations 1999: Report of The Expert Committee on the Diagnosis and Classification of Diabetes Mellitus. Diabetes Care 1999; 22 (Suppl): 1-30.

3. O'Sullivan JB, Mahan CM. Criteria for the oral glucose tolerance test in pregnancy. Diabetes 1964; 13: 278-85

4. Rudge MVC, Calderon IMP, Ramos MD, Abbade JF, Rugolo LMSS. Perinatal outcome of pregnancies complicated by diabetes and by maternal daily hyperglycemia not related to diabetes: a retrospective 10-year analysis. Gynecol Obstet Invest 2000; 50: 108-12.

5. Carpenter MW. Testing for gestational diabetes In: Reece EA, Coustan DR, editors. Diabetes mellitus. In: pregnancy. 2. ed. New York: Churchill Livingstone; 1995. p. 261-76.

6. O'Sullivan JB, Mahan CM, Charles D, Dandrow R. Screening criteria for high-risk gestational diabetic patients. Am J Obstet Gynecol 1972; 116: 895-900.

7. Greenberg LR, Moore TR, Murphy H: Gestational diabetes mellitus: antenatal variables as predictors of postpartum glucose intolerance. Obstet Gynecol 1995; 86: 97-101.

8. Gaudier FL, Hauth FC, Poist M, Corbett L, Cliver SP. Recurrence of gestational diabetes mellitus. Obstet Gynecol 1992; 80: 755-8.

9. McElduff A, Goldring J, Gordon P, Wyndha L. A direct comparison of the measurement of a random plasma glucose and a post 50-g glucose load glucose, in the detection of gestational diabetes. Aust NZ J Obstet Gynecol 1994; 14: 28-30

10. Engelau MM, Aubert RE, Thompson TJ, Herman WH. Screening for NIDDM in nonpregnant adults: a review of principles, screening tests, and recommendations. Diabetes Care 1995; 18: 1606-18.

11. Haffner SM, Rosenthal M, Hazuda HP, Stern MP, Franco LJ. Evaluation of three potential screening tests for diabetes mellitus in biethnic population. Diabetes Care 1984; 7: 34753.

12. West KM, Kalbfleisc JM. Sensitivity and specificity of five screening tests for diabetes in ten countries. Diabetes 1971; 20: 289-96.

13. Sacks DA, Greenspoon JS, Fotheringham N. Could the fasting plasma glucose assay be used to screen for gestational diabetes. J Reprod Med 1992; 37: 907-9.

14. Peterson KA, Petersen AM, Corbett V, Tongen S, Guzman M, Mazze R. Comparison of home glucose monitoring with the oral glucose tolerance test to detect gestational glucose intolerance. J Fam Pract 1994; 39: 558-63.

Recebido em 25 de agosto de 2003

Versão final em 20 de junho de 2005

Aprovado em 26 de julho de 2005
15. Guttorm E. Practical screening for diabetes mellitus in pregnant women. Acta Endocrinol 1974; (Suppl 182): 1124.

16. Catalano PM, Vargo KM, Bernstein IM, Amini SB. Incidence and risk factors associated with abnormal postpartum glucose tolerance in women with gestational diabetes. Am J Obstet Gynecol 1991; (Suppl 165): 914-9.

17. Moses RG. The recurrence rate of gestational diabetes in subsequent pregnancies. Diabetes Care 1996; 19: 1348-50.

18. Garner PR: Glucose metabolism assessment in pregnancy. Clin Biochem 1995; 28: 499-502.

19. Reichelt AJ, Spichler ER, Branchtein L, Nucci LB, Franco LJ Schimidt MI. Fasting plasma glucose is a useful test for the detection of gestational diabetes. Diabetes Care 1998; 21: $1246-9$

20. Bertini-Oliveira AM, Camano LE, Delascio D. Diabetes e gravidez. São Paulo: Sarvier; 1998.

21. O'Sullivan JB. Establishing criteria for gestational diabetes. Diabetes Care 1973; 3: 437-9.

22. Ray R, Heng BH, Lim C, Lim SL. Gestational diabetes in Singaporean women: use of the glucose challenge test as a screening test and identification of high risk factors. Ann Acad Med Singapore 1998; 25: 504-8.

23. Berkowitz GS, Lapinski RH, Wein R, Lee D. Race/ethnicity and other risk factors for gestational diabetes. Am J Epidemiol 1992; 135: 965-73.

24. Sacks DA, Abu-Fadil S, Karten GJ, Forsythe AB, Hackett JR. Screenig for gestational diabetes with the one-hour $50 \mathrm{~g}$ glucose test. Obstet Gynecol 1987; 70: 89-93.

25. Moses RG, Griffiths R, Davi W. Gestational diabetes: do all women need to be tested. Aust NZ J Obstet Gynecol 1995; 35: $387-9$.

26. Jang HC, Cho NH, Jun KB, Oh KS, Dooley SL, Metzger BE. Screening for gestational diabetes mellitus in Korea. Int J Gynecol Obstet 51: 115-22.

27. Solomon CG, Willet WC, Carey VJ, Rich-Edwards J, Hunter DJ, Colditz GA, Stampfer MJ, Speizer FF, Spiegelman D, Manson JF. A prospective study of pregravid determinants of gestational diabetes mellitus. JAMA 1997; 278: 1078-83.

28. Weeks JW, Major CA, Veciana M, Morgan MA. Gestational diabetes: does the presence of risk factors influence perinatal outcome? Am J Obstet Gynecol 1994; 171: 1003-7.

29.Rudge MVC, De Luca LA. Diabete e gravidez. Femina 1981; 9: 463-7.

30.Rudge MVC, Calderon IMP, Ramos MD, Brasil MAM, Peraçoli JC. Comparação de dois métodos de rastreamento do diabete na gestação. Rev Bras Ginecol Obstet 1994; 16 : 203-5.

31.Jekel JF, Elmore JG, Katz DL. Análise bivariada. In: Jekel JF, Elmore JG, Katz DL, editores. Epidemiologia, bioestatística e medicina preventiva. Porto Alegre: Artmed; 1999. p. 155-77. 